HNO 2022 $\cdot 70: 533-539$

https://doi.org/10.1007/s00106-021-01128-8

Angenommen: 11. November 2021

Online publiziert: 4. Februar 2022

() Der/die Autor(en) 2022

\section{Prätherapeutische Dysphagie bei Kopf-Hals-Tumor-Patienten}

\author{
Uta Lehner ${ }^{1}$ Eugen Zaretsky ${ }^{1}$ Almut Goeze ${ }^{1}$. Laura Wermter ${ }^{1}$ Boris A. Stuck ${ }^{2}$. \\ Richard Birk ${ }^{2} \cdot$ Andreas Neff $^{3}$. Ingo Fischer ${ }^{3} \cdot$ Shahram Ghanaati ${ }^{4} \cdot$ Robert Sader $^{4}$. \\ Christiane Hey' \\ 'Abteilung für Phoniatrie und Pädaudiologie, HNO, Universitätsklinikum Gießen und Marburg GmbH, \\ Standort Marburg, Philipps-Universität Marburg, Marburg, Deutschland \\ ${ }^{2}$ Klinik für Hals-, Nasen- und Ohrenheilkunde, Universitätsklinikum Gießen und Marburg GmbH, Standort \\ Marburg, Philipps-Universität Marburg, Marburg, Deutschland \\ ${ }^{3}$ Klinik und Poliklinik für Mund-, Kiefer- und Gesichtschirurgie, Universitätsklinikum Gießen und Marburg \\ $\mathrm{GmbH}$, Standort Marburg, Philipps-Universität Marburg, Marburg, Deutschland \\ ${ }^{4}$ Klinik für Mund-, Kiefer-, Plastische Gesichtschirurgie, Universitätsklinikum Frankfurt/Main, Goethe- \\ Universität Frankfurt/Main, Frankfurt/Main, Deutschland
}

Hintergrund: Sowohl der Schluck- als auch der Ernährungsstatus bei Kopf-HalsTumor(KHT)-Patienten nach einer onkologischen Therapie sind gut untersucht. Prätherapeutisch werden sie aber selten thematisiert, obwohl diese den Erfolg einer onkologischen Therapie nachhaltig beeinflussen können.

Ziel der Arbeit: Ziel dieser Arbeit ist die systematische Erfassung des Schluck- und Nutritionsstatus von KHT-Patienten vor Beginn einer onkologischen Therapie. Material und Methoden: Bei 102 Patienten wurden zur objektiven Erfassung des Schluckvermögens endoskopisch die Penetration/Aspiration via PA-Skala (PAS), die Oralisierungseinschränkung (Functional Oral Intake Scale, FOIS) und die Versorgungsrelevanz (VRS) erhoben. Die subjektive Einschätzung des Schluckvermögens erfolgte via Fragebogen gEAT-10 ("German EAT-10"), die orientierende Erfassung des Nutritionsstatus via Body-Mass-Index (BMI). Schluckvermögen und BMI wurden uni- und multivariat auf mögliche Einflussfaktoren geprüft.

Ergebnisse: Auffällige PAS-, FOIS- und VRS-Werte wurden bei $\leq 15 \%$ der Patienten festgestellt. Der BMI war häufiger zu hoch als zu niedrig. Das objektiv erfasste Schluckvermögen war v.a. vom Tumorstadium abhängig und korrelierte mittelstark mit gEAT-10. Der gEAT-10-Gesamtscore war auffällig. Der Nutritionsstatus war von Patientengeschlecht und VRS abhängig.

Schlussfolgerung: Prätherapeutisch zeigte sich bei der Mehrzahl der Patienten keine Dysphagie oder Malnutrition. Ein auffälliges Schluckvermögen war mit höheren Tumorstadien assoziiert, eine Malnutrition mit weiblichem Geschlecht und Versorgungsrelevanz. Nichtsdestotrotz sollte hinsichtlich moderner onkologischer Therapie der Schluck- und Nutritionsstatus bei KHT-Patienten bereits prätherapeutisch systematisch erfasst werden, um ein optimales Patienten-Outcome zu erzielen.

\title{
Schlüsselwörter
}

Schluckstörung · Body-Mass-Index · EAT-10 · Aspiration · Malnutrition

Die einschneidenden Funktionsstörungen infolge einer Kopf-Hals-Tumor-Erkrankung können die Lebensqualität und soziale Teilhabe der Betroffenen erheblich beeinträchtigen. Schluckstörungen führen darüber hinaus zu einer Steigerung von Morbidität und Morta- lität und sind damit nicht nur für den Patienten, sondern auch von sozioökonomischem Interesse. Eine frühzeitige systematische objektive wie subjektive Erfassung einer möglichen Schluckstörung vor onkologischem Therapiebeginn könnte den onkologischen Krank- 


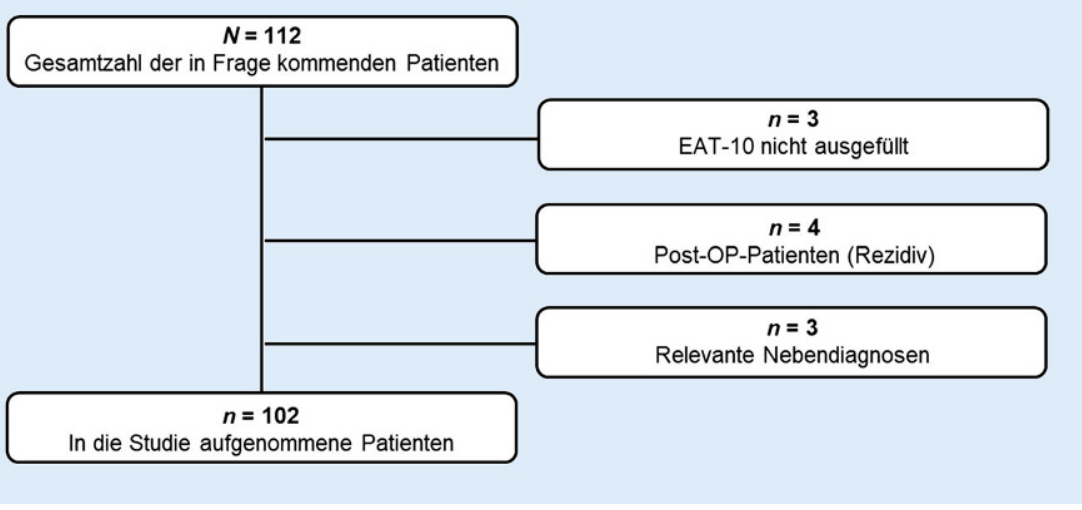

Abb. 1 ॥ Patienteneinschlussdiagramm. EAT Eating Assessment Tool. OP Operation

\section{heitsverlauf entscheidend beeinflussen und ist daher Gegenstand der vorliegen- den Studie.}

Schluckstörungen infolge einer onkologischen Therapie bei Kopf-Hals-Tumor-Patienten sind gut belegt [1-3], während die Ermittlung ihrer Prävalenz vor Beginn einer onkologischen Therapie weniger im wissenschaftlichen Fokus steht $[4,5]$. Dabei bestehen Hinweise, dass mögliche Folgekomplikationen, wie v.a. die Malnutrition, Auswirkungen auf den Verlauf einer onkologischen Kopf-Hals-Tumor-Erkrankung nehmen. So identifizierten Tsai et al. [6] eine präoperative Malnutrition als unabhängigen Prädiktor für postoperative Komplikationen bei Kopf-Hals-Tumor-Patienten. Chang et al. [7] belegten einen signifikanten Zusammenhang zwischen niedrigem prätherapeutischem Body-Mass-Index (BMI) und frühem Versterben eines Patienten mit Kopf-Hals-Tumor noch während der onkologischen Behandlung. Dabei wird die Malnutrition einer onkologischen Erkrankung meist auf deren konsumierenden Charakter zurückgeführt [8], konnte jedoch von Jager-Wittenaar et al. [9] und Ottoson et al. [10] als relevante Folge einer Schluckstörung nachgewiesen werden.

Diese Studien legen die Notwendigkeit nahe, bereits prätherapeutisch den Dysphagiestatus eines Kopf-Hals-Tumor-Patienten systematisch und detailliert zu erfassen, um die peri- und posttherapeutische Versorgung von Schluckstörungen sicherzustellen und angepasst an den individuellen Bedarfbesser planen zu können. Mithilfe der instrumentellen Diagnostik können dabei nicht nur die Penetration/Aspiration und Oralisierungseinschränkung, sondern auch die sich aus diesen beiden Kernproblemen ergebende Versorgungsrelevanz erhoben werden.

Dabei bezieht die moderne onkologische Versorgung den Patienten mit seinen Interessen aktiv in den Therapieprozess mit ein, sodass neben der objektiven Erhebung einer Schluckstörung auch die subjektive Einschätzung des Patienten in Bezug auf Umfang und Schwere der Dysphagie von Relevanz ist. Hierfür eignet sich der international anerkannte Fragebogen EAT-10 [11], der als gEAT-10 in deutscher für Kopf-Hals-Tumor-Patienten validierter Übersetzung vorliegt [12]. Er besteht aus insgesamt zehn 5-Punkte-Likertskalierten Fragen ( 0 „kein Problem“ und 4 "schwerwiegendes Problem“), die neben spezifischen Symptomen auch soziale und emotionale Aspekte einer Schluckstörung berücksichtigen.

Ziel der vorliegenden Arbeit war die systematische Untersuchung des Schluckvermögens bei Kopf-Hals-Tumor-Patienten, v.a. der Penetration/Aspiration, Oralisierungseinschränkung und Versorgungsrelevanz, vor Beginn einer onkologischen Therapie. Zusätzlich erfolgte die orientierende Erfassung des Ernährungsstatus mittels BMI. Zudem war von Interesse, inwieweit Tumorgröße, -lokalisation, Patientenalter und -geschlecht Einfluss auf die Schluckfähigkeit nehmen und inwiefern die objektive Erfassung der Schluckfunktion mit der subjektiven Patienteneinschätzung übereinstimmt.

\section{Methodik}

Für diese Studie liegen Ethikvoten aus zwei Kliniken vor: Universitätsklinikum Frankfurt/Main (AZ 240/10) und Universitätsklinikum Marburg (AZ 63/15).

\section{Stichprobe}

Die Durchführung der vorliegenden Studie erfolgte prospektiv im Zeitraum von August 2015 bis Mai 2021 in der HalsNasen-Ohrenheilkunde und Mund-, Kiefer- und Gesichtschirurgie des Universitätsklinikums Marburg sowie in der Mund-, Kiefer-, Plastischen Gesichtschirurgie des Universitätsklinikums Frankfurt/Main.

Für die Studie wurden Patienten mit einem neu diagnostizierten Mundhöhlen-, Oropharynx-, Larynx- bzw. Hypopharynxtumor, Stadium I-IV (UICC, Union International Contre le Cancer), vor Beginn einer onkologischen Therapie rekrutiert. Inkludiert wurden Patienten im Alter $\geq 18$ Jahre, mit schriftlicher Einwilligungserklärung und vollständigem Datensatz. Ausschlusskriterien bildeten das Vorliegen eines Kopf-Hals-Tumor-Rezidivs sowie weitere schluckbeeinträchtigende Erkrankungen wie z.B. Encephalitis disseminata.

Für die Studie wurden 112 Patienten rekrutiert, wovon 10 aufgrund nicht zutreffender Ein- bzw. Ausschlusskriterien exkludiert wurden (• Abb. 1).

Insgesamt wurden demnach $102 \mathrm{~Pa}$ tienten, 18 weiblich $(17,6 \%), 84$ männlich $(82,4 \%)$, im Alter von im Mittel $62,6 \pm 8,5$ Jahren in die Studie aufgenommen (• Tab. 1).

Zwischen Männern und Frauen bestanden keine Altersunterschiede laut MannWhitney-U-Test $(p>0,05)$.

\section{Untersuchungsablauf}

Alle Patienten erhielten eine FEES ${ }^{\oplus}$-Diagnostik (flexible endoskopische Evaluation des Schluckvorgangs) nach Langmore-Protokoll zur objektiven Erfassung möglicher Penetration/Aspiration, Oralisierungseinschränkung und Versorgungsrelevanz einer Schluckstörung, inklusive der anatomisch-physiologischen Untersuchung, der Schluckfunktionsprüfung sowie der Überprüfung von Haltungspositionen und Schluckmanövern [13, 14]. Für die Schluck- 
Tab. 1 Patientencharakteristika der Studienpopulation (Alter, Tumorstadium nach UICC und -lokalisation)

\begin{tabular}{|l|l|}
\hline $\begin{array}{l}\text { Patienten- } \\
\text { charakteristika }\end{array}$ & \begin{tabular}{l} 
Gesamt- \\
stichprobe \\
\cline { 2 - 2 }
\end{tabular} \\
\hline Tumorstadium
\end{tabular}

funktionsprüfung wurden standardisiert flüssige und pürierte (in den Volumina 2, 5,10 und $20 \mathrm{ml}$ ) sowie feste Konsistenzen eingesetzt, sofern das Schluckvermögen des Patienten dies erlaubte.

Die Schweregradeinteilung der Penetration/Aspiration erfolgte mit der Penetrations-Aspirations-Skala (PAS) nach Rosenbek $([15,16]$; - Infobox 1), die der Oralisierungseinschränkung mit der Functional Oral Intake Scale (FOIS) nach Crary ([17]; - Infobox 2). Die Originalreihenfolge der FOIS wurde für statistische Analysen invertiert und damit an die Reihenfolge der PAS adaptiert [18]. Eine schwerwiegende Penetration bestand bei PAS 4-5, Aspiration ab PAS $\geq 6$, eine therapierelevante Oralisierungseinschränkung $\mathrm{ab} F \mathrm{FOIS} \geq 4$. Die Versorgungsrelevanz (VRS) einer Schluckstörung wurde auf Basis der ersten beiden Kardinalsymptome erschlossen und wie folgt festgelegt: $P A S \geq 4$ bzw. FOIS $\geq 4$.

Die Erfassung der subjektiven Wahrnehmung des Schluckvermögens aus Sicht der Patienten erfolgte über den Fragebogen gEAT-10 (German Eating Assessment Tool, • Tab. 4).

\section{Statistische Analysen}

Als Erstes erfolgte die deskriptiv statistische Aufbereitung des Auftretens von PAS, FOIS und VRS sowohl für die Gesamtstichprobe als auch für einzelne Tumorstadi-

Tab. 2 Absolute und relative Häufigkeit der Penetration/Aspiration (PAS), Oralisierungseinschränkung (FOIS) und der versorgungsrelevanten Schluckstörung (VRS) sowie Mittelwerte (M) vom Body-Mass-Index (BMI) und vom gEAT-10-Gesamtscore, abhängig von den vier möglichen Einflussfaktoren

\begin{tabular}{|c|c|c|c|c|c|}
\hline Einflussfaktoren & PAS & FOIS & VRS & BMI & gEAT-10 \\
\hline & $n(\%)$ & $n(\%)$ & $n(\%)$ & $M+S D$ & $M+S D$ \\
\hline \multicolumn{6}{|l|}{ Tumorstadium } \\
\hline I & $0(0,0)$ & $0(0,0)$ & $0(0,0)$ & $28,6 \pm 4,5$ & $1,0 \pm 1,7$ \\
\hline II & $0(0,0)$ & $0(0,0)$ & $0(0,0)$ & $24,9 \pm 4,0$ & $5,6 \pm 8,6$ \\
\hline III & $1(4,5)$ & $0(0,0)$ & $2(9,1)$ & $26,6 \pm 4,9$ & $5,2 \pm 6,7$ \\
\hline IV & $7(11,3)$ & $7(11,3)$ & $13(21,0)$ & $25,2 \pm 5,1$ & $10,4 \pm 12,0$ \\
\hline \multicolumn{6}{|l|}{ Tumorlokalisation } \\
\hline Mundhöhle & $1(5,3)$ & $0(0,0)$ & $1(5,3)$ & $27,7 \pm 5,8$ & $5,4 \pm 8,2$ \\
\hline Oropharynx & $3(6,7)$ & $2(4,4)$ & $6(13,3)$ & $24,8 \pm 4,0$ & $8,6 \pm 11,1$ \\
\hline Hypopharynx/Larynx & $4(10,5)$ & $5(13,2)$ & $8(21,1)$ & $25,7 \pm 5,4$ & $9,1 \pm 11,2$ \\
\hline \multicolumn{6}{|l|}{ Alter (Jahre) } \\
\hline $41-60$ & $2(4,9)$ & $1(2,4)$ & $3(7,3)$ & $25,2 \pm 5,3$ & $6,4 \pm 8,7$ \\
\hline$>60$ & $6(9,8)$ & $6(9,8)$ & $12(19,7)$ & $26,0 \pm 4,7$ & $9,4 \pm 11,7$ \\
\hline \multicolumn{6}{|l|}{ Geschlecht } \\
\hline Weiblich & $1(8,3)$ & $0(0,0)$ & $1(5,6)$ & $23,9 \pm 4,9$ & $9,4 \pm 10,6$ \\
\hline Männlich & $7(5,6)$ & $7(8,3)$ & $14(16,7)$ & $26,0 \pm 4,9$ & $7,9 \pm 10,7$ \\
\hline
\end{tabular}

en, -lokalisationen, Altersgruppen und Geschlecht, des Weiteren die des BMI-Werts, Gewichtsverlusts (aktuelles Gewicht in Bezug auf Normalgewicht vor Erkrankungsbeginn) und des gEAT-10-Gesamtscores.

Der Zusammenhang zwischen PAS, FOIS, VRS sowie BMI und den möglichen Einflussfaktoren Tumorstadium, -lokalisation, Patientenalter und -geschlecht wurde univariat untersucht. Dies erfolgte mit nichtparametrischen Testverfahren aufgrund des ordinalen Skalenniveaus von PAS, FOIS und VRS.

Der Zusammenhang zwischen PAS, FOIS, VRS sowie BMI und UICC-Tumorstadien wurde mittels Spearman-Korrelationen berechnet.

Die Werteverteilung von PAS, FOIS, VRS und BMI wurde abhängig von der Tumorlokalisation zuerst mit Kruskal-Wallis$\mathrm{H}$-Tests geprüft. Die anschließenden paarweisen Vergleiche einzelner Tumorlokalisationen erfolgten mit Mann-Whitney-UTests, ebenso wie die Untersuchung der Werteverteilung abhängig vom Patientengeschlecht.

Die Zusammenhangsstärke zwischen Patientenalter sowie PAS, FOIS, VRS und BMI wurde via Spearman-Korrelationen geprüft.
Darüber hinaus wurde der BMI mit allen drei Parametern (PAS, FOIS, VRS) korreliert.

Die Analyse möglicher Einflussfaktoren auf PAS, FOIS und VRS erfolgte mit drei linearen Regressionen (Methode „Einschluss"), mit den unabhängigen Variablen Tumorstadium und -lokalisation sowie Patientengeschlecht und -alter. Die Aussagekraft des jeweiligen Gesamtmodells wurde mit einer ANOVA bestimmt und die erklärte Varianz mit dem korrigierten Nagelkerkes $\mathrm{R}^{2}$. Die Einflussstärke der einzelnen überprüften Faktoren wurde mit standardisierten Beta-Koeffizienten ermittelt.

In einer weiteren linearen Regression wurden BMI als abhängige, dagegen Tumorstadium, -lokalisation, Patientengeschlecht und -alter sowie VRS als unabhängige Variablen eingesetzt.

Die Zusammenhangsanalyse der objektivierbaren (PAS, FOIS und VRS) mit der subjektiv empfundenen Einschätzung des Schluckvermögens (gEAT-10-Gesamtscore und -Einzelitems) erfolgte via Spearman-Korrelationen. Für die erste gEAT-10Frage (subjektiver Gewichtsverlust) wurde zusätzlich eine Spearman-Korrelation mit dem objektiv erfassten Gewichtsverlust berechnet. 


\section{Infobox}

Die 8-Punkte-Penetrations-Aspirations-

Skala nach Rosenbek [15]

- 1 Material dringt nicht in den Luftweg ein

- 2 Material dringt in den Luftweg ein, verbleibt oberhalb der Stimmlippen und wird aus dem Luftweg ausgestoßen

- 3 Material dringt in den Luftweg ein, verbleibt oberhalb der Stimmlippen und wird nicht aus dem Luftweg ausgestoßen

- 4 Material dringt in den Luftweg ein, kontaktiert die Stimmlippen und wird aus dem Luftweg ausgestoßen

- 5 Material dringt in den Luftweg ein, kontaktiert die Stimmlippen und wird nicht aus dem Luftweg ausgestoßen

- 6 Material dringt in den Luftweg ein, passiert bis unter die Stimmlippen und wird in den Larynx hinein oder aus dem Luftweg ausgestoßen

- 7 Material dringt in den Luftweg ein, passiert bis unter die Stimmlippen und wird nicht aus der Trachea ausgestoßen, trotz Bemühung

- 8 Material dringt in den Luftweg ein, passiert bis unter die Stimmlippen, und es wird keine Bemühung zum Ausstoßen unternommen

\section{Ergebnisse}

Von allen 102 Patienten wiesen 6 (5,9\%) eine schwerwiegende Penetration und 8 $(7,8 \%)$ eine Aspiration auf. Eine therapierelevante Oralisierungseinschränkung fand sich bei $7(6,9 \%)$, eine versorgungsrelevante Schluckstörung bei $15(14,7 \%)$ der 102 Patienten.

Der prätherapeutische BMI-Wert lag im Mittel bei $25,6 \pm 4,9 \mathrm{~kg} / \mathrm{m}^{2} \quad(14,53-$ $\left.39,31 \mathrm{~kg} / \mathrm{m}^{2}\right)$. Gemäß Weltgesundheitsorganisation (WHO) [19] zeigten von den 102 Patienten 6 (5,8\%) ein Untergewicht $\left(<18,5 \mathrm{~kg} / \mathrm{m}^{2}\right), 43(42,2 \%)$ ein Normalgewicht $\left(18,5-24,9 \mathrm{~kg} / \mathrm{m}^{2}\right), 31 \quad(30,4 \%)$ eine Präadipositas $\left(25,0-29,9 \mathrm{~kg} / \mathrm{m}^{2}\right), 17$ $(16,4 \%)$ eine Adipositas Grad I (30$\left.34,9 \mathrm{~kg} / \mathrm{m}^{2}\right)$ und $5(4,9 \%)$ eine Adipositas Grad II $\left(35,0-39,9 \mathrm{~kg} / \mathrm{m}^{2}\right)$.

Der ungewollte Gewichtsverlust betrug im Mittel 2,8 $44,5 \mathrm{~kg}(0-20 \mathrm{~kg})$. Von den 102 Patienten wiesen $12(11,8 \%)$ einen ungewollten Gewichtsverlust $\leq 5 \mathrm{~kg}$ auf, 20 $(19,6 \%)>5-10 \mathrm{~kg}, 5(4,9 \%)>10-20 \mathrm{~kg}$. Damit zeigten insgesamt 25 Patienten (24,5\%) einen Gewichtsverlust von über $5 \mathrm{~kg}$. Bei 65 der 102 Patienten (63,7\%) blieb das Gewicht stabil.
Infobox 2

Die Functional Oral Intake Scale nach Crary [17] (invertiert)

- 1 Vollständig orale Diät ohne Einschränkungen

- 2 Vollständig orale Diät mit verschiedenen Konsistenzen, ohne spezielle Zubereitung, aber mit Einschränkungen spezifischer Lebensmittel

- 3 Vollständig orale Diät mit verschiedenen Konsistenzen, aber spezieller Zubereitung oder Kompensation

- 4 Vollständig orale Diät mit einer Konsistenz

- 5 Sondenabhängig mit konsistenten Versuchen oraler Aufnahme von Nahrung oder Flüssigkeiten

- 6 Sondenabhängig mit minimalen Versuchen oraler Gabe von Nahrung oder Flüssigkeiten

- 7 Nichts über den Mund

Der gEAT-10-Gesamtscore variierte zwischen 0 und 40 und lag im Durchschnitt bei $8,2 \pm 10,6$. Die Werteverteilung von PAS, FOIS, VRS, BMI und gEAT-10, abhängig von möglichen Einflussfaktoren, ist - Tab. 2 zu entnehmen.

Das Tumorstadium korrelierte schwach bis mittelstark mit allen drei Parametern: $\operatorname{PAS}(\rho=0,284 ; p=0,004), \operatorname{FOIS}(\rho=-0,340$; $p<0,001)$ und VRS $(\rho=0,350 ; p<0,001)$, nicht jedoch mit dem BMI $(p>0,05)$.

Die Penetration/Aspiration, Oralisierungseinschränkung, Versorgungsrelevanz sowie BMI unterschieden sich nicht signifikant abhängig von der Tumorlokalisation ( $p s>0,05$ ), auch nicht bei den paarweisen Vergleichen einzelner Tumorlokalisationen, außer dass bei Patienten mit einem Larynx-/Hypopharynxkarzinom auffälligere PAS-Werte erzielt wurden als bei solchen mit einem Mundhöhlenkarzinom $(Z=-2,2 ; p=0,029)$.

Der Zusammenhang zwischen Patientengeschlecht bzw. -alter und PAS, FOIS, VRS sowie BMI war nicht signifikant (ps $>0,05$ ).

Es zeigten sich schwache, aber signifikante Spearman-Korrelationen zwischen BMI und PAS $(\rho=-0,273 ; p=0,006)$, FOIS $(\rho=-0,257 ; p=0,009)$ und VRS $(\rho=-0,278 ; p=0,005)$.

In den linearen Regressionen mit PAS, FOIS und VRS als abhängige Variablen wurde lediglich das Tumorstadium durchgehend als relevanter Einflussfaktor detektiert (- Tab. 3): je höher das Tumorstadium, desto höher die Skalenwerte. Niedrigere BMI-Werte waren mit dem weiblichen Geschlecht und auffälligeren Werten in der Versorgungsrelevanz assoziiert.

Auffälligere Befunde in gEAT-10 waren durchgehend signifikant mit auffälligeren PAS-, FOIS- und VRS-Skalenwerten sowie mit niedrigeren BMI-Werten assoziiert (• Tab.4). Die Stärke einzelner Korrelationen variierte zwischen minimal und mittelstark. Zusammenhänge zwischen gEAT10 und PAS lagen ausnahmslos niedriger als solche mit VRS und vor allem FOIS.

Der tatsächliche Gewichtsverlust war bei Patienten mit auffälligeren Befunden im gEAT-10-Item 1 stärker ausgeprägt $(\rho=0,610 ; p<0,001)$.

\section{Diskussion}

Patienten mit einem Kopf-Hals-Tumor vor Beginn einer onkologischen Therapie zeigten ein seltenes Vorkommen einer objektivierbaren Schluckstörung in Bezug auf Penetration/Aspiration, Oralisierungseinschränkung und Versorgungsrelevanz. Wenn, so fand sich eine Schluckstörung v.a. bei Patienten mit UICC-Stadien III und IV. Damit zeigte sich bereits prätherapeutisch eine Tendenz des objektiv ermittelten Schluckvermögens, die in früheren Studien bei Patienten nach onkologischer Therapie nachgewiesen wurde: je höher das Tumorstadium, desto ausgeprägter die Schluckstörung [20-22].

Unterschiede im Schweregrad einer Schluckstörung je nach Tumorlokalisation waren minimal ausgeprägt. Patienten mit einem Larynx- bzw. Hypopharynx-Karzinom zeigten etwas häufiger Auffälligkeiten hinsichtlich Aspiration/Penetration und einer therapierelevanten Schluckstörung als Patienten mit einem Mundhöhlenkarzinom, was ob der Lokalisation einen zu erwartenden Befund darstellt [23]. Patientenalter und -geschlecht waren dagegen mit allen drei Parametern (PAS, FOIS, VRS) nicht signifikant assoziiert.

Die Patienten beurteilten ihr Schluckvermögen subjektiv mit einem durchschnittlichen gEAT-10-Gesamtscore von 8 als auffällig, legt man das von Belafsky et al. [11] definierte Cut-off-Kriterium $\geq 3$ zugrunde. Es zeigte sich dabei eine hohe Übereinstimmung der objektivierbaren Schluckbeeinträchtigung mit der sub- 
Tab. 3 Werteverteilung der Penetrations-Aspirations-Skala (PAS), Functional Oral Intake Scale (FOISumgepolt) und der Skala derversorgungsrelevanten Schluckstörung (VRS) sowie Body-Mass-Index (BMI) in Abhängigkeit von möglichen Einflussfaktoren:vier lineare Regressionen (mit standardisierten Beta-Koeffizienten 6 )

\begin{tabular}{|l|l|l|l|l|}
\hline & PAS & FOIS & VRS & BMI \\
\hline Gesamtmodell: $F$ & $3,99^{* *}$ & $3,97^{* *}$ & $4,88^{* *}$ & $4,42^{* *}$ \\
\hline Korrigiertes Nagelkerkes $R^{2}$ & 0,11 & 0,11 & 0,13 & 0,15 \\
\hline Tumorstadium: $6 / T$ & $0,271 / 2,824^{* *}$ & $0,298 / 3,096^{* *}$ & $0,311 / 3,286^{* *}$ & $-0,018 /-0,185$ \\
\hline Tumorlokalisation: $6 / T$ & $0,208 / 2,165^{*}$ & $0,122 / 1,269$ & $0,188 / 1,982^{*}$ & $-0,081 /-0,839$ \\
\hline Alter: $6 / T$ & $0,104 / 1,083$ & $0,140 / 1,463$ & $0,132 / 1,394$ & $0,142 / 1,497$ \\
\hline Geschlecht: $6 / T$ & $-0,088 /-0,915$ & $-0,075 /-0,778$ & $-0,090 /-0,956$ & $-0,214 /-2,278^{*}$ \\
\hline VRS: $6 / T$ & - & - & - & $-0,382 /-3,784^{* * *}$ \\
\hline Konstante: $T$ & $-0,942$ & $-1,004$ & $-1,082$ & $6,615^{* * *}$ \\
\hline *** $p<0,001 ;{ }^{* *} p<0,01 ;{ }^{*} p<0,05$ & \multicolumn{3}{l}{} \\
\hline
\end{tabular}

Tab. 4 Zusammenhang zwischen gEAT-10 und der Penetrations-Aspirations-Skala (PAS), Functional Oral Intake Scale (FOIS umgepolt), der Skala der versorgungsrelevanten Schluckstörung (VRS) sowie Body-Mass-Index (BMI): Spearman-Korrelationen

\begin{tabular}{|c|c|c|c|c|}
\hline gEAT-10 & PAS & FOIS & VRS & BMI \\
\hline Item 1: Mein Schluckproblem führte zu Gewichtsverlust & $0,315^{* *}$ & $0,495^{* * *}$ & $0,419^{* * *}$ & $-0,489^{* * *}$ \\
\hline $\begin{array}{l}\text { Item 2: Mein Schluckproblem beeinträchtigt meine Möglichkeit, zum Essen auszuge- } \\
\text { hen }\end{array}$ & $0,364^{* * *}$ & $0,628^{* * *}$ & $0,519^{* * *}$ & $-0,364^{* * *}$ \\
\hline Item 3: Das Schlucken von Flüssigkeit erfordert besondere Anstrengung & $0,351^{* * *}$ & $0,390^{* * *}$ & $0,400^{* * *}$ & $-0,395^{* * *}$ \\
\hline Item 4: Das Schlucken von fester Nahrung erfordert besondere Anstrengung & $0,419^{* * *}$ & $0,612^{* * *}$ & $0,574^{* * *}$ & $-0,384^{* * *}$ \\
\hline Item 5: Das Schlucken von Tabletten erfordert besondere Anstrengung & $0,389^{* * *}$ & $0,585^{* * *}$ & $0,509^{* * *}$ & $-0,459^{* * *}$ \\
\hline Item 6: Schlucken ist schmerzhaft & 0,149 & $0,369^{* * *}$ & $0,267^{* *}$ & $-0,396^{* * *}$ \\
\hline Item 7: Die Freude am Essen ist durch mein Schlucken beeinträchtigt & $0,384^{* * *}$ & $0,488^{* * *}$ & $0,454^{* * *}$ & $-0,334^{* * *}$ \\
\hline Item 8: Wenn ich schlucke, bleibt mir Nahrung im Hals stecken & $0,376^{* * *}$ & $0,489^{* * *}$ & $0,464^{* * *}$ & $-0,359^{* * *}$ \\
\hline Item 9: Ich huste, wenn ich esse & $0,218^{*}$ & $0,389^{* * *}$ & $0,326^{* * *}$ & $-0,327^{* * *}$ \\
\hline Item 10: Schlucken ist anstrengend & $0,301^{* *}$ & $0,538^{* * *}$ & $0,429^{* * *}$ & $-0,380^{* * *}$ \\
\hline gEAT-10-Gesamtscore & $0,379^{* * *}$ & $0,582^{* * *}$ & $0,402^{* * *}$ & $-0,456^{* * *}$ \\
\hline
\end{tabular}

jektiven Einschätzung der Patienten, die bereits in einigen früheren Studien für die prätherapeutische Phase demonstriert wurde $[24,25]$.

Der Ernährungszustand war mit einem durchschnittlichen BMI von $26 \mathrm{~kg} / \mathrm{m}^{2}$ gemäß WHO-Einteilung eher als präadipös zu werten. Nur knapp $6 \%$ aller Patienten wiesen eine Malnutrition auf, $25 \%$ einen ungewollten Gewichtsverlust oberhalb von $5 \mathrm{~kg}$, was trotz des klaren Überwiegens eines normalgewichtigen oder sogar adipösen Ernährungszustands einen Hinweis möglicher Malnutritionsgefährdung bildet [26]. Dieses Ergebnis sollte daher ob der Tragweite hinsichtlich der Beeinflussung des onkologischen Therapieoutcomes [27] mit geeigneten Erfassungstools prospektiv untersucht werden.

Während sich der BMI als unabhängig von Tumorlokalisation, -stadium und Patientenalter erwies, bestand ein signi- fikanter Zusammenhang zum einen zur Schwere einer Schluckstörung, gemessen an der Versorgungsrelevanz, zum anderen zum Patientengeschlecht: Höhere BMIWerte fanden sich v.a. bei Patienten mit keiner oder nur geringer Beeinträchtigung des Schluckens und beim männlichen Geschlecht.

Die hier mittels Regressionsanalysen untersuchten Faktoren hinsichtlich einer möglichen Einflussnahme auf Penetration/ Aspiration, Oralisierungseinschränkung, Versorgungsrelevanz und BMI ergaben eine Varianzerklärung von maximal $15 \%$, was bedeutet, dass weitere wichtige Einflussfaktoren existieren, die hier nicht berücksichtigt wurden.

Vergleicht man die hier untersuchte $\mathrm{Pa}$ tientenpopulation mit den 2019 vom Robert Koch-Institut (RKI) publizierten Zahlen zu Patienten mit einem Kopf-Hals-Tumor [28], so ist die hier verwendete Stichpro- be repräsentativ in Bezug auf Tumorlokalisation und Patientenalter, nicht jedoch in Bezug auf das Geschlechtsverhältnis. Hier sind die Männer in Relation zu den RKI-Daten überrepräsentiert, was die Vergleichbarkeit zwischen Männern und Frauen in der aktuellen Studie zusätzlich reduziert. Da generell immer deutlich mehr Männer von einer Kopf-Hals-Tumorerkrankung betroffen sind, sind per se diskrepante Ergebnisse zwischen dieser Art von uni- und multivariaten Analysen zu erwarten. Um eine Generalisierung der vorliegenden Studienergebnisse vornehmen zu können, bedarf es daher einer Vergrößerung der Studienpopulation. Eine größere Fallzahl ließe dann auch die separierte Analyse des Schluckvermögens für die unterschiedlichen Tumorlokalisationen (z.B. Hypopharynx/Larynx) zu.

Nichtsdestotrotzzeigen die hier präsentierten prospektiv erhobenen Studiener- 
gebnisse, dass Kopf-Hals-Tumor-Patienten bereits prätherapeutisch sowohl objektiv als auch subjektiv Schluckstörungen ebenso wie Auffälligkeiten des Ernährungszustands aufweisen können. Diese gilt es, in der weiteren onkologischen Behandlungsplanung bzw. im Verlauf einer onkologischen Therapie zu berücksichtigen, um weitere Folgekomplikationen zu verhindern bzw. den Patienten trotz aller Einschränkungen das Maximum an Lebensqualität zu ermöglichen.

\section{Fazit für die Praxis}

- Trotz hier niedriger Prävalenzrate einer versorgungsrelevanten Schluckstörung und eines Untergewichts bestehen Hinweise darauf, dass v.a. Patienten mit höheren Tumorstadien und Larynx-/ Hypopharynx-Karzinomen bereits prätherapeutisch bzgl. ihres Schluckvermögens und Nutritionsstatus beeinträchtigt sind.

- Auch wenn nur sehr wenige Patienten bei Diagnosestellung einen BMI unter der Norm aufweisen, berichten $25 \%$ über einen ungewollten Gewichtsverlust von $>5 \mathrm{~kg}$.

- Daher sollten alle Kopf-Hals-Tumor-Patienten v.a. höheren Tumorstadiums hinsichtlich Schluck- und Nutritionsstatus systematisch bereits vor Beginn der onkologischen Therapie untersucht werden.

- Das Ziel: eine personenzentrierte Optimierung der peritherapeutischen onkologischen Versorgung von Kopf-Hals-Tumor-Patienten.

\section{Korrespondenzadresse}

\section{Eugen Zaretsky}

Abteilung für Phoniatrie und Pädaudiologie, HNO, Universitätsklinikum Gießen und Marburg GmbH, Standort Marburg, PhilippsUniversität Marburg

Baldingerstr. 1, 35043 Marburg, Deutschland zaretsky@staff.uni-marburg.de

Funding. Open Access funding enabled and organized by Projekt DEAL.

\section{Einhaltung ethischer Richtlinien}

Interessenkonflikt. U. Lehner, E. Zaretsky, A. Goeze, L. Wermter, B.A. Stuck, R. Birk, A. Neff, I. Fischer, S. Ghanaati, R. Sader und C. Hey geben an, dass kein Interessenkonflikt besteht.

Für diese Studie liegen Ethikvoten aus zwei Kliniken vor: Universitätsklinikum Frankfurt/Main (AZ 240/10) und Universitätsklinikum Marburg (AZ63/15).
Open Access. Dieser Artikel wird unter der Creative Commons Namensnennung 4.0 International Lizenz veröffentlicht, welche die Nutzung, Vervielfältigung, Bearbeitung, Verbreitung und Wiedergabe in jeglichem Medium und Format erlaubt, sofern Sie den/die ursprünglichen Autor(en) und die Quelle ordnungsgemäß nennen, einen Link zur Creative Commons Lizenz beifügen und angeben, ob Änderungen vorgenommen wurden.

Die in diesem Artikel enthaltenen Bilder und sonstiges Drittmaterial unterliegen ebenfalls der genannten Creative Commons Lizenz, sofern sich aus der Abbildungslegende nichts anderes ergibt. Sofern das betreffende Material nicht unter der genannten Creative Commons Lizenz steht und die betreffende Handlung nicht nach gesetzlichen Vorschriften erlaubt ist, ist für die oben aufgeführten Weiterverwendungen des Materials die Einwilligung des jeweiligen Rechteinhabers einzuholen.

Weitere Details zur Lizenz entnehmen Sie bitte der Lizenzinformation auf http://creativecommons.org/ licenses/by/4.0/deed.de.

\section{Literatur}

1. Greco E, Simic T, Ringash J, Tomlinson G, Inamoto $Y$ (2018) Dysphagia treatment for patients with head and neck cancer undergoing radiation therapy: a meta-analysis review. Int J Radiat Oncol Biol Phys 101(2):421-444

2. Pezdirec M, Strojan P, Hocevar Boltezar I (2019) Swallowing disorders after treatment for head and neck cancer. Radiol Oncol 53(2):225-230

3. Carmignani I, Locatello LG, Desideri I et al (2018) Analysis of dysphagia in advancedstage head-and-neck cancer patients: impact on quality of life and development of a preventive swallowing treatment. Eur Arch Otorhinolaryngo 275(8):2159-2167

4. Martín Villares C, Tapia Risueño M, San Román Carbajo J, Fernández Pello ME, Domínguez Calvo J (2003) Pretreatment dysphagia in patients with advanced head and neck cancer. Nutr Hosp 18(5):238-242

5. Patterson J, Wilson JA (2011) The clinical value of dysphagia preassessment in the management of head and neck cancer patients. Curr Opin Otolaryngol Head Neck Surg 19(3):177-181

6. Tsai YT, Lai CH, Huang TH et al (2020) Association of malnutrition with postoperative complication risk after curative surgery for oral cancer: observational study. Medicine 99(52):e23860

7. Chang P-H, Yeh K-Y, Huang J-S et al (2013) Pretreatment performance status and nutrition are associated with early mortality of locally advanced head and neck cancer patients undergoing concurrent chemoradiation. Eur Arch Otorhinolaryngol 270(6):1909-1915

8. Talwar B, Donnelly R, Skelly R, Donaldson M (2016) Nutritional management in head and neck cancer:United Kingdom national multidisciplinary guidelines. J Laryngol Otol 130(S2):S32-S40

9. Jager-Wittenaar H, Dijkstra PU, Vissink A et al (2011) Malnutrition in patients treated for oral or oropharyngeal cancer-prevalence and relationship with oral symptoms: an explorative study. Support Care Cancer 19(10):1675-1683

10. Ottosson S, Lindblom U, Wahlberg P et al (2014) Weight loss and body mass index in relation to aspiration in patients treated for head and neck cancer: a long-term follow-up. Support Care Cancer 22(9):2361-2369

11. Belafsky PC, Moudabed DA, Rees CJ et al (2008) Validity and reliability of the eating assessment tool (EAT-10). Ann Otol Rhinol Laryngol 117(12):919-924

12. Zaretsky E, Steinbach-Hundt S, Pluschinski P et al (2018) Validierung der deutschen Version des Eating Assessment Tool bei Kopf-Hals-TumorPatienten. Laryngorhinootologie 97(7):480-486

13. Langmore SE, Schatz K, Olsen N (1988) Fiberoptic endoscopic examination of swallowing safety: a new procedure. Dysphagia 2(4):216-219

14. Hey C, Pluschinski P, Stanschus S et al (2011) A documentation system to save time and ensure proper application of the fiberoptic endoscopic evaluation of swallowing $\left(\mathrm{FEES}^{\circledR}\right)$. Folia Phoniatr Logop 63(4):201-208

15. Rosenbek JC, Robbins JA, Roecker EB et al (1996) A penetration-aspiration scale. Dysphagia 11(2):93-98

16. Hey C, Pluschinski P, Zaretsky Y et al (2014) Penetrations-Aspirations-Skala nach Rosenbek. Validierung der deutschen Version für dieendoskopische Dysphagiediagnostik. HNO62(4):276-281

17. Crary MA, Carnaby Mann GD, Groher ME (2005) Initial psychometric assessment of a functional oral intake scale for dysphagia in stroke patients. Arch Phys Med Rehabil 86(8):1516-1520

18. Hey C, Lange BP, Aere Cet al (2013) Predictability of oral and laryngopharyngeal function for aspiration and limitation of oral intake in patients after surgery for head and neck cancer. Anticancer Res 33(8):3347-3354

19. Weltgesundheitsorganisation (2000) Obesity: preventing and managing the global epidemic. Report of a WHO consultation. World Health Organ Tech RepSer 894:1-253

20. Klingelhöffer C, Obst A, Ettl T et al (2019) Severe postoperative dysphagia as an early predictor for decreased overall survival in patients with oral cancer. J Craniomaxillofac Surg 47(9):1363-1369

21. Amin JD, Rodriggs T, Weir KA et al (2021) Prospective evaluation of swallowing symptoms in human papillomavirus-associated oropharynx cancer. Dysphagia. https://doi.org/10.1007/s00455-02110249-9

22. Goeze A, Zaretsky E, Lehner U et al (2021) Postoperative Dysphagieprävalenz bei Kopf-HalsTumor-Patienten im akutstationären Setting. Laryngorhinootologie. https://doi.org/10.1055/a1528-7584

23. Starmer H, Edwards J (2019) Clinical decision making with head and neck cancer patients with dysphagia. Semin Speech Lang 40(3):213-226

24. Arrese LC, Carrau R, Plowman EK (2017) Relationship between the Eating Assessment Tool-10 and objective clinical ratings of swallowing function in individuals with head and neck cancer. Dysphagia 32(1):83-89

25. Bofill-Soler N, Guillen-Sola A, Marco E et al (2021) Is EAT-10 useful to assess swallowing during the chemo-radiotherapy phase in patients with head and neck cancer? A pilot study. Ann Otol Rhinol Laryngol 130(7):689-698

26. Einarsson S, Laurell G, Tiblom Ehrsson Y (2020) Mapping the frequency of malnutrition in patients with head and neck cancer using the GLIM criteria for the diagnosis of malnutrition. Clin Nutr ESPEN 37:100-106

27. Kapała A, Surwiłło-Snarska A, Jodkiewicz $M$, Kawecki A (2021) Nutritional care in patients with head and neck cancer during chemoradiotherapy (CRT) and bioradiotherapy (BRT) provides better 
compliance with the treatment plan. Cancers (Basel) 13(11):2532

28. Robert Koch-Institut (2019) Krebs in Deutschland für 2015/2016, 12. Aufl. RobertKoch-Institut, Berlin

\section{Pre-treatment dysphagia in head-and-neck cancer patients}

Background: The swallowing and nutritional status of head-and-neck cancer patients after oncological therapy have been extensively researched. However, the same topics are seldom scrutinized before the onset of oncological therapy, although they can influence treatment success in the long term.

Objective: This study focusses on a systematic assessment of swallowing function and nutritional status in head-and-neck cancer patients prior to oncological therapy.

Materials and methods: In 102 patients, penetration/aspiration (PA scale), limitations of oral intake (Functional Oral Intake Scale, FOIS), and the need for further intervention (NFI) were endoscopically assessed to objectively quantify swallowing function. The subjective evaluation of swallowing function was carried out with the gEAT-10 (German EAT-10) questionnaire, nutritional status was assessed by body mass index (BMI). Possible impact factors for swallowing function and BMI were analyzed by univariate and multivariate methods.

Results: PAS, FOIS, and NFI values were abnormal in $\leq 15 \%$ of patients. BMI was more often too high than too low. Objectively assessed swallowing functions depended predominantly on tumor stage and showed moderate correlations with gEAT-10. The latter mostly yielded a "fail" result. The nutritional status depended on the patients' biological sex and NFI.

Conclusion: In the pre-treatment setting, neither dysphagia nor malnutrition were found in most patients. Impaired swallowing was associated with higher tumor stages, malnutrition with female sex and NFI. A systematic pre-treatment assessment of swallowing and nutritional status in head-and-neck cancer patients appears necessary for modern oncological therapy and optimal patient outcome.

\section{Keywords}

Deglutition disorders $\cdot$ Body mass index $\cdot$ EAT-10 $\cdot$ Aspiration $\cdot$ Malnutrition 